\title{
Use of the Internet as a Means for the Development of Sexual Identity and Social Intolerance
}

\author{
Soler Alomà J* \\ UPF-Tecnocampus, Spain
}

*Corresponding author: Jordi Soler Alomà, UPF-Tecnocampus, NAVARRO 3, 4rt - 1a, Spain, Tel: 660884554; Email: jsoler@tecnocampus.cat

\section{Research article}

Volume 4 Issue 4

Received Date: December 09, 2021

Published Date: December 31, 2021

DOI: $10.23880 /$ phij-16000215

\section{Abstract}

The development of sexuality is a problem for contemporary society, since the mechanisms through which the biological calendar expresses itself include the Internet. Society is not yet ready to assume a natural fact that "violates morality."

Keywords: Development; Sexual; Structure; System; Moral; Internet; Intolerance

\section{Preface}

In the new "space of states" that the internet represents, a good part (which progressively increases) of the processes that configure our life takes place. Among the processes that happen on the internet there are, with increasing prevalence, those that contribute to the development of personal identity, of which what we designate as sexual identity is a part. The two quotes that are adduced below are a sample of the growing interest that the phenomenon of new forms of expression of eroticism has in the development of the so-called sexual identity for development sciences, a constitutive part, as has already been mentioned, of personal identity.

“Children's sexuality has been a taboo subject in many societies, but its existence is undeniable. A variety of sexual behaviours such as masturbation are evident starting in early childhood. Sexual games become frequent in middle childhood, especially among girls. Cross-cultural and crossspecies evidence suggests that sexual desire emerges around middle childhood, along with adrenarche, which is the early phase of puberty. However, sexuality in childhood does not always signify sexual orientation in later developmental stages. In addition, different biosocial factors appear to contribute to the development of sexuality during childhood. Taking a developmental systems perspective, this chapter synthesizes existing evidence and points out areas for future research. The substantive gaps involve examining coacting and bidirectional processes in sexuality development, focusing on sexual agency, and recording dynamic change. Transforming research methods and fostering societal recognition of children's sexuality may be critical for not only advancing research on sexuality development in childhood, but also improving children's well-being." Li, Gu [1].

"To examine adolescent sexuality on the Internet, the chapter begins by presenting a co-construction framework connecting youths' online behaviour to their development. Then we describe the characteristics of online environments that support sexual exploration and sexuality online. Finally, we describe the ways that adolescents engage in online sexual exploration in the service of their sexual development: searching for information about sexuality and sexual health, constructing and presenting sexual selves online, cybersex (sexual chatting between two or more individuals), and accessing sexually explicit content. We show that the Internet presents both opportunities and challenges to youths as they engage in these kinds of online explorations in the service of their sexual development." Smahel, David; SubrahmanyaM, Kaveri [2].

In the previous quotes we can see two ways of dealing with the same issue. The first goes directly to the core of the object of study, without prejudice or safeguards, whereas 
the second, despite the fact that it directly mentions "what is the matter", observes at a "prudent" distance what it intends to investigate, as if it was a highly polluting substance, thus giving off the feeling that the matter, in reality, "is foreign to the authors".

In our case, we will adopt the same type of approach to the object of study as that of the first quote: a direct approach, carried out on empirical material and without prejudice or safeguards, because we understand that facing observable facts, one should not proceed as when speculating on the intricate phenomena of quantum physics. Therefore, to carry out this study, a selection of videos corresponding to the different ways in which eroticism is expressed on the networks has been viewed and analysed, both of recordings made by the users themselves and uploaded and of stream recordings made in real time. Only in this way could firsthand information be obtained, to confer a robustness to the study that went beyond speculation based on statistics, reference books, or the work of colleagues who may have used both media.

\section{Introduction}

The process known as development of sexual identity, which is part of the development of personal identity, is a complex research object, as is the study of any bio-psychosocial process. Needless to say, the concept of development includes all the stages it goes through. For this reason, many scholars use an approach based on conventionally established "milestones" that indicate at what exact moment each event related to this phenomenon occurs. This "milestionian" approach has been surpassed by the most advanced researchers in the field, who have discovered that a dynamic and systemic approach is much more productive and consistent, and that it takes into account, in addition to biological processes (whose classic approach, by the way, has already been overcome), all other factors that influence the process under study [vid supra].

\begin{tabular}{|c|c|c|c|c|c|c|}
\hline \multicolumn{7}{|c|}{ Milestonian approach } \\
\hline Event & Time & Event & Time & Event & Time & Event \\
\hline Birth & $8 \mathrm{a} \rightarrow$ & Stage 1 & $10 \mathrm{a} \rightarrow$ & Stage 2 & $12 \mathrm{a} \rightarrow$ & Stage 3 \\
\hline
\end{tabular}

Table 1: This type of approach assigns specific dates to each event based on purely biological criteria (own elaboration).

\section{Systemic dynamic approach}

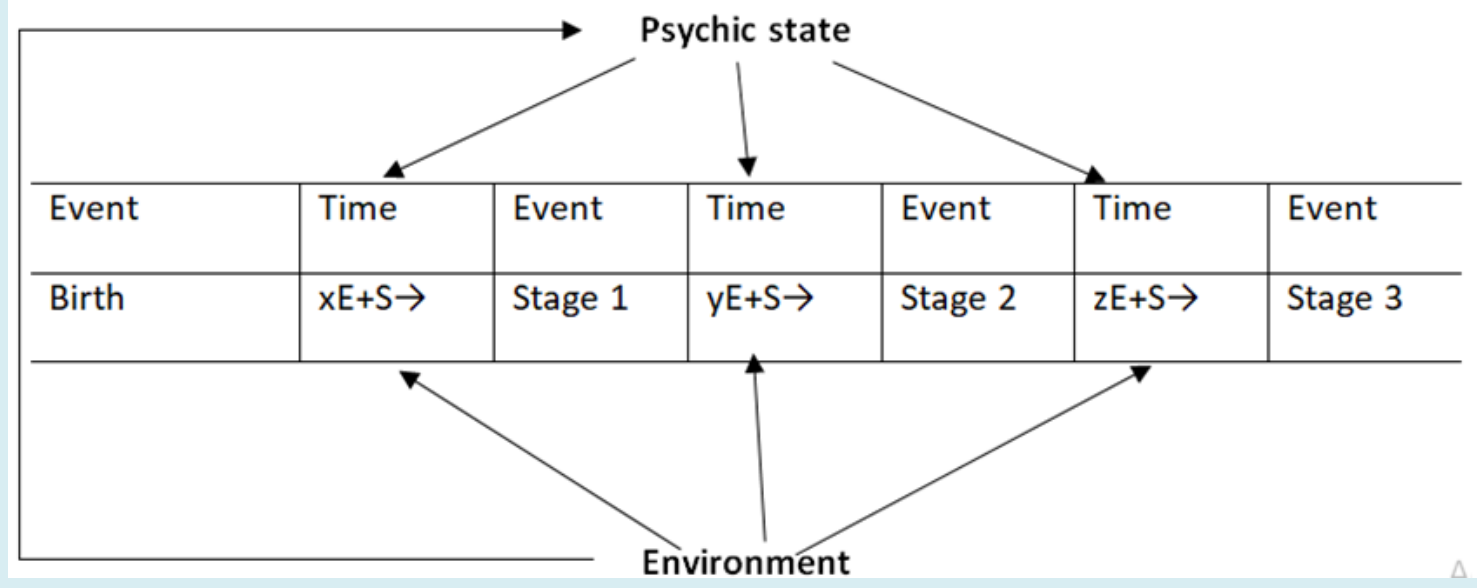

Figure 1: The systemic-dynamic approach takes into account the influences of the environment on the psyche, and of the latter on the phenotype, as well as the direct influences that the environment exerts on it. $(E=$ environment; $\mathrm{S}=\mathrm{mental}$ state [own elaboration]).

In reviewing the literature on this subject, we find that many researchers complain about the lack of material in general and the lack of reliable material in particular. All of them agree to attribute this shortage to the "taboo" factor. For instance, at the beginning of his article on the emergence of sexuality, $\mathrm{Gu} \mathrm{Li}$ [1] laments that, in developmental 
sciences, research on child sexuality is practically nonexistent. He attributes this neglect to the aura of taboo that surrounds this topic. It is both a social and a parental problem: in general, parents refuse to talk about sexuality with their sons and daughters (or do so "out of obligation" and superficially); society does not recognize the ability of children to have "sexual sensations", under the fallacy of a certain epiphenomenon called "innocence" which, according to a certain "urban mythology" entirely assumed by public administrations, could be affected. Let us specify the concepts a bit: "innocence" presupposes its opposite, "guilt" and, therefore, in the same way that, in advance, children are not "guilty" of anything, nor can they be "innocent" of anything; such alleged argument of "innocence itself" is nothing more than a fallacy to hide the deep generalized ignorance about what the integral development of the human being is, of which the process we study is part. Under the pretence of "protecting innocence" what is being done is to hide one's own ignorance. Another fallacy, which is not consciously assumed as such, although it is constantly wielded with other words, is to pretend that the child's scarcity of information, in contrast to the abundance of information that an adult supposedly possesses, is actually something like "essence" of said "innocence" and that, furthermore, it must be preserved... who can be so foolish as to pretend to keep someone plunged into ignorance under the subterfuge of "protecting their innocence"? Pre-adolescents and adolescents cannot be protected by restricting their rights and freedoms and preventing their access to information; on the contrary, if what is really sought is their protection, this can only be achieved by protecting their rights and freedoms and providing them with the means to exercise them freely and safely. On the other hand, the (in our way of seeing) hasty, superficial and not very reflective attitude of public administrations towards this phenomenon, which causes negative changes in the context, has the pernicious effect of displacing those who are supposed to have the obligation to safeguard towards spaces that are less safe than conventional means [vid supra].

This myth of "innate innocence" is a childish reaction to another myth: that of "original sin", which Adam and Eve committed in paradise (note that because of Eva [a woman]) and which had two immediate consequences, in addition of the expulsion: the feeling of shame in the face of nudity (which they did not have before, since they were naked in paradise) and the transmission to the entire human species of the sin originated by Eve. Consequently, according to the Holy Bible and the different religions that subscribe to it, children are not innocent, since they are born with original $\sin$ in tow. But since we equate children to angels, to us they are innocent and "therefore" do not have sex. This myth of "innate innocence", which undermines the scientific nature of research on the development of sexual identity, and that tries to ignore that there are biological processes with their own calendar that must be externalized and, in the case of humans, socialized, is dismantled by serious research (Bancroft, 2003; Graaf and Rademakers, 2006; Diamond, Bonner and Dickenson, 2015; Sandfort and Rademakers, 2000) [1] in which it is found that, although sexual relations are rare among children, they can participate in other types of sexual activities, such as kissing, hugging or playing with the genitals, and that according to Graaf and Rademakers (ibid), Diamond, et al., these early sexual experiences are an integral part of healthy development and have a lasting positive impact in later development periods, as has been demonstrated in the results of the studies with control groups, and as demonstrated in the study (which lasted 40 years) of the sexual evolution of a group of students by Borneskog, Catrin, et al.. That is why psychobiology is interested in this facet of personal development, since a systemic approach to such processes reveals that the drives originated in mechanisms of sexual development promote their expression in the environment (in the case of humans, as said before, their "socialization"); the result of this interaction will notably influence various factors of the subjects' personality, and may even define their sexual identity.

There are several speculative trends as to when, during a person's life, the so-called "sexuality" appears. In addition to this, said concept is either not defined in any of the publications that deal with the matter, or it is defined in a vague way or, to get out of trouble, it is resorted to a mere biological explanation. It seems that, as with "dark matter", no one can determine exactly what "sexuality" consists of. That is why it may be preferable to refer to this human property as eroticism, since this term does not have all the connotations that the term "sex" has and that have been attached to it by the different "morals" that have precautions regarding this matter. These connotations are alien to the thing itself and certainly come from ancestral prejudices and "social taboos" (we will expand on this later); by such substitution, we can avoid, at the very least, the methodological error of using an a priori concept (that is, taken for granted).

One of the opinions held by some psycho-neurologistsis that the "sexual" phenomenon is associated with the development of the hippocampus; consequently, minors of a certain age (not specified) would lack this human "property" [3]. It is clear that, in this matter, Mario Bunge, in an attitude far away from that which distinguishes him as one of the best philosophers of science in history, even though he is partially correct, omits three quite relevant facts: $a$ ) in the human society "sex", in addition to being a reproductive factor of the species, is a cultural element, b) "sexual" properties do not appear in all parts of the world at the same age and the moment of their appearance differs greatly depending 
on the type of society (there are even people who remain "asexual" throughout life) and, therefore, the appearance of the property "sex" looks no less phenotypic than genotypic and $c$ ) there are other psychobiological (or simply biological) hypotheses better founded than that of the hypothalamus (as will be seen later). At the other end of "when does the thing appear" exists psychoanalysis, with Freud at the head [4], for whom "sexuality" is something innate and, furthermore, believes that all adult problems come from repression of what he calls "sexual drives" during childhood. It has been demonstrated that, despite rare exceptions of a pathological nature, Freud was (as in almost everything else) wrong, although it must be granted that he was right that the repression of the expression of sexual drives (which, remember, are the mechanisms through which the organism manifests itself in its development) is the cause of many problems in relation to personal development, and, very especially, during the stage called "adolescence." On a truly scientific side, VanderLaan, DP, Wong, WI (and their collaborators) argue with foundation that the birth of "sexuality" is linked to the development or "awakening" of the adrenal gland that takes place between 6 to 8 years, and is related to increased production of adrenal androgens such as dehydroepiandrosterone, dehydroepiandrosterone sulphate, and androstenedione, processes also involved in the growth of pubic hair. A couple of years later, the gonadal glands begin to mature, promoting the production of gonadal hormones such as testosterone and oestradiol) $[5,6]$, which promote the growth of the ovaries and testes, as well as the breasts and genitals; This is the empirically most plausible and scientifically well-founded option, without prejudice to the participation, in the process, of the hippocampus, constituting a system in which this would be a "reinforcement" component. This evidence places the phenomenon studied at an earlier age, a quite pertinent fact that, however, can make mentalities that are not very open to novelty uncomfortable. In any case, these "milestonian" approaches (as Gu Li aptly nicknames them) do not coincide with what is observed when the fear of leaving the library is lost and we move beyond the stereotypes fixed in this matter, which are reproduced by repeated readings of the same reference works. Already in some research work carried out at the beginning of the century is revealed that, in this matter, the interaction with the context can be as determining (or, in some cases, even more) for the phenotype as is the genotype [7]. Reveal that recent advances in epigenetics show that context can regulate gene expression [1]. Be that as it may, it is unquestionable that the context, whether it is positive (or benign) or negative (or toxic) has a significant influence on individual behaviour. To what extent this can be expressed phenotypically remains to be determined, but it is not the core of our topic.

Being consistent with all of the above, society nor cannot ignore the responsibility of assuming that the Internet, which is the new context that mediates practically everything in our lives, is also a new mechanism that, through different forms of eroticism's expression, mediates the development of sexual identity neither cannot avoid the duty to study the subject seriously and without interfering with the rights and freedoms of the interested parties since, in the way that this matter is treated by entities and public administrations, it gives the impression that the interested parties are not treated as subjects of law. Certainly, no one can deny that the issue (not for intrinsic reasons, but extrinsic) is thorny and that an open mind and a certain amount of courage are needed to face the issue openly.

The Internet has made available to us real samples of "sexual behaviour" in the pre-adult stages, something that has never existed until now. Several studies (i.e. Smahel and Subrahmanyam, 2014) [2] confirm that teens and tweens use digital media to construct and present their sexual identity, and, for obvious reasons, they do so independently of parents (and researchers). It would be a proverbial naivety to believe that the development of science and technology (what we call "new technologies"), which modifies all aspects of our life to a degree that, only a few years ago, we would have believed impossible, would not also affect to a factor as intimately human as eroticism and the construction of one's sexual identity (it is clear that it intervenes in many other facets of the personality, but they are not the object of this study and have already been extensively treated in the ad hoc bibliography). Consequently, both scientific research and public policies should take into account this "singularity" in the "event horizon" of the Internet and make an effort to update. Although the facts do not change (the exploration and construction of sexual identity), the mechanisms through which it unfolds change. Thus, with regard to the public administration, it is not enough to install computers in their headquarters: it is (to use a metaphor) "open the windows wide so that fresh air can get in".

In the educational institutional world there is a certain unanimity that must be given information about "sexuality" to students. On the other hand, there is no unanimity on how to do it, so that the information provided is, in general, biased and poorly structured; so much so that, even, the students themselves complain about this fact and, therefore, it is not surprising that they contrast the information in other sources, the main one being the Internet (as already mentioned). The issue is usually not different in families: there are fathers and mothers who share that the knowledge of "sexuality" by their sons and daughters is something positive and even progressist (which, objectively, is equivalent to considering progressist the explanation of the functioning of the digestive system) but who are horrified at the possibility that these (especially if they are daughters) can express and 
contrast their eroticism through the use of the Internet: this is where "progressivism" could reach! It seems that society has forgotten that, when the Internet did not exist, sexual curiosity and "erotic drives" already existed at an early age, and were manifested in other ways (for example, playing "doctors" - a name that, by the way, has its explanation) and in other spaces, such as the toilets and changing rooms of educational centers, the home rooms or the ones in houses of relatives, friends or neighbours, or any place outdoors (a typical phrase was "you show me the yours and I show you the mine ") away from furtive glances, in addition to publications classified as "erotic" or "pornographic" by the establishment. Consequently, perhaps it would be better to try to understand the causes and forms of manifestation of this phenomenon (the expression of eroticism on the Internet and its role in the development of sexual identity), and find the best way to act, where appropriate, without undermining the rights and freedoms of those affected, instead of adopting an inquisitive attitude (as has always been done in when facing what is either not understood, or "cannot be accepted", as it happened, and still does, with homosexuality and other "anomalies"). All the scientific studies consulted in this work coincide in an undeniable fact: the place where adolescents and pre-adolescents learn the most about what we call "sex" consists of is the Internet [see bibliography].

This study goes in the direction of making an analysis as objective as possible of what the phenomenon of adolescent (teen) and pre-adolescent (tween) erotic expression on the Internet consists of, focused specifically on the female space, as it is, by far the most prevalent; although meticulous studies argue, with good reason, biological causes of female precociousness (i.e. $\mathrm{Gu} \mathrm{Li}, 2020$ ) [1], the search for some psycho-sociological explanation would be a necessary complement to the holistic understanding of the phenomenon since, although the genotype "facilitates" the structure, the phenotype is what "builds" the system. It should be mentioned that the different sexual liberation movements (avoiding the use of acronyms, since within the movement itself there is no agreement on this matter) have facilitated the gradual presence of both adolescents and nonbinary adults in this phenomenon.

This research focuses on three platforms selected for their significance in the evolution of the analysed phenomenon: YouTube, Stickam and Periscope. In addition to this introduction, a descriptive part will be developed through $a$ ) in which essential information about the platform analysed will be provided and a taxonomy of the different intervening agents will be completed (the authors, followers and the society), as well as a characterization of their roles and the typologies found in the different subgroups); b) a conclusive part, where the most objective possible assessment of the phenomenon will be carried out and, where appropriate, proposals for solution, channelling and optimization (and, surely, correction of drift) will be provided; also, as an annex, c) a historical part, which goes from the beginning of the object of this study on YouTube to its current stage in m-apps, passing through various streaming platforms. [For more technical and detailed information on the different platforms studied, see, below, the "file" of each one in the corresponding links].

It is not a secret that the nature of this study can arouse susceptibilities, an unfortunately "natural" effect that is caused by the conditioning factors of our culture, still underdeveloped in this matter (a fact that is denounced, tacitly or explicitly, by most part of the researchers).

As already mentioned, the study has been carried out (withoutforgetting the mostadvanced research on the subject, of which there is a sample in the bibliography) directly on the viewing of videos from YouTube and recordings of "streams" that were broadcasted through the studied platforms. This empirical procedure compensates, in our opinion, the weak point that afflicts this type of studies, which, based on interviews, more or less reliable surveys, statistics and articles by colleagues, do not have the empirical character (in the sense of verifiable) that requires a scientific investigation, especially when the investigated object can be examined with the naked eye (the only necessary equipment is a computer). Since some of the conceptual results achieved through the aforementioned (and "questioned") methodology are quite interesting, they have been incorporated into the conceptual baggage that supports this work, which is, to put it somehow, the necessary complement to such thoughtful investigations.

Both in the streams and in the recorded videos you can see the indicators of the presence of "sexual activity" that swarm by the different studies on the subject, such as: flushed face, thick breathing, sweating, pupil dilation, self-absorption and blurring eyes.

\section{Internet as a "Space of States"}

\section{YouTube: A "Self-Showcase"}

In order not to extend almost ad infinitum, we limit the beginning of the phenomenon that this research deals with in the early days of YouTube [vid infra]. This medium becomes a platform on which, from the beginning, some users begin to "post" videos (recorded by themselves) in different attitudes or "performances" qualifying as "erotic", without implying nudity beforehand (later, the typological detail and other relevant aspects will be entered). Thus, channels are created, managed by the authors themselves, which reach a large number of followers and "likes". Coinciding with the monetization stage of the videos that followed the placement 
of advertising banners, there are cases in which the parents themselves act as "managers" for their daughters, filming, editing the "footage" and configuring the channels. There are two paradigmatic cases of this variety: those of $M P$ and $M B$ (for obvious reasons, the names are omitted), two "erotic superstars" (one tween and the other teen) of YouTube. Unfortunately, the ultra-conservative sectors of North American society, "scandalized" by this awakening of eroticism on the internet, unleashed a "holy war" (a sort of anti-pantie jihad) against "heresy". It is not a secret that these guilds are related to powerful lobbies (when they are not in themselves). Following complaints from some significant entities, the company (YouTube), without giving any explanation, decided to wholesale suppress all profiles of "minors" with content that could be subject to any type of "anti-pantie" censorship, so that even those channels that dealt with gymnastics, yoga, and similar were wiped off the map. Soon, YouTube created a section for minors, but with the requirement of parental supervision, thus restricting the freedom of expression and the rights of the interested parties; predictably, it has resulted in a resounding failure. Later on we will deal with the counterproductive consequences that, throughout this period, this reaction (in the pejorative sense of the term) has had precipitous, not meditated and guided by mere image criteria (even when YouTube presumed to be a public service) , a reaction that, like a row of dominoes, has spread to most of the known analogue platforms [8-12].

\section{Typological Considerations}

Putting it briefly, there are different modalities within this range of YouTube videos. There are recordings that are manifestly improvised, but there are others that have been studied and practiced before filming and, even, there are those that have a script, costumes, makeup, choreography and post-production (editing in real time or, later, of the footage). As can be seen, all the videos coincide in several aspects: their authors delight in what they do; they make use of all their skills to make them enjoyable; at the time of completion, the faces of its protagonists express enormous satisfaction, in addition to the feeling of a certain self-realization. We can also verify and disprove, through this valuable material, some of the hypotheses developed through surveys and interviews, as well as certain topics that truffle the manuals on sexual development. This set of essential features will be complemented, later, with a detailed psychological analysis of each of the most significant videos.

Since it has been observed that there are different types of video that coincide in their characteristics, they can be classified into categories:

\section{1: According to the format}

a) Improvisation: It is a type of video in which the protagonist records her evolutions, provided with greater or lesser erotic intentionality, without prior preparation (that is, there are no previous elements such as a script or a wardrobe selection). The author allows her underwear to be seen, at her discretion, and when she does, she pretends that she is not aware of it but she shows signs of wanting the viewer to know that she pretends not to be aware of it.

b) Real-time editing: This type of video has the same format as the previous one, except for a non-trivial detail: the author stops the recording, whenever she considers it appropriate, to see how each shot has gone. In this way, she can gradually modify her performance based on the obtained feedback.

c) Pre-production: This is a type of video with all the elements of a "professional" production: script, costumes, makeup, choreography, etc. Thus, the content of the video recording has been previously designed.

d) Post-production: This type can come from categories "a" or "c", but with the incorporation of the editing of the footage. In this way, a selection of the best shots can be made for the final product; add effects; add soundtrack (or modify it); enter text; etc.

\section{2: According to content}

a) Crafts and toys: In this type of video the protagonist appears doing some kind of craft or playing with an object; as she does so, she offers clearly intentional visuals of her underwear and/or, depending on the type of outfit she wears, her pecs.

b) Gymnastics, yoga challenges and similar: As can be deduced from the heading, this modality involves the display of underwear and/or pectorals during the practice of gymnastic exercises, complex yoga postures (usually in pairs), sets of ladder panels with rings and ropes, martial arts (usually improvised), and "obstacle courses" through the room.

c) Choreographies: This variety, which is what allows the protagonists to express their eroticism more explicitly and openly, is largely based on dance, and includes their own choreographies or those of famous artists. Before the advent of censorship, some of these videos had their music removed for infringing a copyright.

d) Routines: Here, the protagonist simply films what she does each day when she gets out of bed or goes to sleep (these are the most prevalent types of "routine", although there are "routines" of any activity, no matter how irrelevant it is); obviously, it is done in such a way as to express a (variable) degree of eroticism.

As has already been stated previously, the videos are usually filmed by their protagonists, although they may also have been filmed by third parties, who may be friends, relatives, or the father or mother (or both). In the case of 
videos filmed by third parties, it may happen that there is no erotic intention on the part of the protagonists, simply because they have not been informed of being recorded. In general, these types of films (especially if they are made by parents) are about very young children. From a certain age they prefer to be the ones who carry out their filming (or be filmed by friends). However, there are cases of complicity (not in a pejorative sense) between parents and children in the expression of their eroticism, as happened in the aforementioned videos (i.e. M.P. and M.B.).

In the case-by-case study, the essential details of each video will be analysed, studying the evolution of the different "states" and interpreting the facial and body expressions that correspond to each of them. A comparative table will also be prepared to highlight both the common and the specific elements. Finally, an attempt will be made to find something like a "measurement standard" for "erotic intensity".

\section{Stickam: The Rise of "Streamers"}

As YouTube succumbed to a tornado of censorship, a powerful platform for live broadcast (real time) or "streaming" called Stickam appeared [vid infra]. This website allowed users to register for this service from the age of 14 , who could create their profiles and channels and broadcast live through the platform. The main difference between Stickam and YouTube was that the former offered the possibility of direct ("live stream", a service that YouTube incorporated later in a rather precarious way). A large number of followers or fans could be connected to live broadcasts [we will call them that for simplicity, whether they were officially or not] and each person could create their own channel from which to broadcast their live broadcasts with the possibility of interacting with their fans (regulars or sporadic). As had happened on YouTube, some customers (mostly girls) used the medium as a channel not only for communication but also for expressing their eroticism and, predictably, a large number of YouTube users migrated to Stickam, since to that platform the devastating hurricane of censorship had not yet arrived. The interaction in real time with the followers was a feedback that, contrary to what happened with the "likes" and fans of the YouTube videos and channels, could influence the development of the streams (both because of what it does performances as well as fan restraint). In this way, in certain channels some followers proposed challenges or dares to some of the streamers (as said above, this was already happening on YouTube, with the exception that were the protagonists of the videos who made a list of challenges, which, written on pieces of paper and put in a container, they drew at random; or they "faced" the challenge or dare that was in fashion, such as the famous ice bucket challenge). Some of these challenges were childish (such as standing upright or throwing a bucket of cold water on top) and others consisted, for example, of "removing" items of clothing. If a fan (or occasional user) bothered a streamer, she could mute him and even kick him off the channel for a while or permanently. It was not difficult to infer that some streamers would show their nudity and even play erotically or with their "sexuality". This phenomenon is very important and clearly marks the fundamental difference between the two analysed platforms: in Stickam there were, a priori, no limits to erotic expression, so that (in the group we studied) the streamers could "get carried away", without any hindrance, for their impulses and to experience their sexuality in a free and natural way in interaction with the public. It is important to highlight the fact of free naturalness, since it is the opposite of what happens in chats established as a business (18+), in which the protagonists clearly show signs of boredom, sadness or stress due to financial need.

In articles on the development of sexual identity, when dealing with the phenomenon of the relationship of tweens and teens with the Internet, the authors usually refer mainly to access to information and the supposed "dangers" of the Internet; some tiptoe over the topic of streams, but within a smorgasbord in which Facebook, Instagram and YouTube are the same fuzzy thing. As far as we know, no one has bothered to study in depth the phenomenon of erotic expression that can be observed in the aforementioned YouTube videos, nor to analyse the content of the Stickam or Periscope streams, when they are, by far, the most interesting and important part of the phenomenon that is the object of research. If what is intended to analyse is the development of sexual identity in contemporary times (and not in the seventeenth century) and, furthermore, the transcendental role of the Internet in this process is clearly assumed... shouldn't attention be paid to the fact that that a large number of Internet users utilize platforms such as those studied in our work to express their eroticism? Shouldn't this phenomenon be analysed with scientific rigor, in depth and without equivocal omissions? Shouldn't the significance of this phenomenon for the supposed object of study, namely the development of sexual identity in the 21st century, be thoroughly investigated? Well, it seems that in the XXI century, when it comes to the sexuality of this population area, all the "anti" alarms go off and all the demons associated with the matter emerge. In this way, it is quite difficult for an objective researcher to be able to access the empirical material with which to base their hypotheses and develop their arguments through contrasted data (and not through bibliographic references unrelated to this matter). Let's be clear: for an adolescent girl, to experiment with her body is something natural and necessary, and there is nothing wrong with it; there is no demon hiding in her vagina and her breasts are not possessed; no exorcism is required. If this same teenager wants to do it on her channel, to share her experience with her fans, we will not find Lucifer or Beelzebub anywhere (although perhaps 
some fundamentalist "sees" them). If we are really interested in this phenomenon, what we must do is, first of all, shake off all the baggage of prejudices with which we come "from the factory" and analyse the facts from a scientific perspective: that is, observe the whole context, including the possible "moral" categories that apply to the case. In the study of this phenomenon, the object cannot be isolated from its context, since this context has a significant influence (and basically to the detriment of the researcher's own criteria) in the research, as has been observed in all the articles consulted, which have the same bias at this point. Consequently, the critical analysis of the criteria with which society and public institutions handle this type of phenomenon is not only urgent but absolutely necessary, because, in addition to the damage caused to young people by erroneous public policies, they are also a ballast for free investigation, thereby generating a feedback loop.

\section{Periscope: A Multifaceted Medium}

At the same time that Stickam was closed, its space was occupied by a new platform, called Periscope (owned by Twitter [vid infra]), which offers the same features as Stickam but with the addition of being suitable for use with any device mobile phone equipped with a camera, which considerably facilitates "live streams" from anywhere and at any time, with a video quality far superior to that of Stickam. Periscope also served as a transmission mechanism for many spontaneous and even professional reporters, who recorded events and broadcasted them through this medium; there are even TV channels that broadcast their programming through this platform. Like Stickam, it was also possible to chat with an unlimited number of users, which was an incentive for streamers for whom breaking the current record was a challenge in itself. Despite the fact that streamers could restrict access to their channel and allow it only to their trusted fans, quantity prevailed, so to speak, over quality. Having the highest number of visitors was the main objective. However, those who actively intervened with the highest prevalence in the chat were usually the unconditional fans, as had happened in Stickam.

The types of performance that could be seen in Periscope were, for example, girls parties (more or less naughty); sensual to erotic dances (which, occasionally, could end in "sex games"); improvised stripteases and $Q \& A$ (a modality that did not involve nudity but could affect very private matters; answering or not certain questions and linking or not the answers to an agreed type of activity depended entirely on the streamers) and, of course, there were also the different modalities of dares and challenges, which, as in previous platforms, could involve the gradual elimination of clothing items.
In Periscope, however, there was never the degree of intimacy that had characterized Stickam, and which meant, for streamers, to be "among friends." Perhaps that is why the type of performance was not as personal and intimate as in the previous platform, in which a streamer could share with her fans her "secret" erotic fantasies to the extent that she felt sufficiently comfortable. However, since in the Russian idiosyncrasy sex is not a taboo (unlike in the "western world"), for some of the Russian streamers, the majority in Periscope, the concept of intimacy of a performance was irrelevant compared to the possible number of visitors achievable.

It should be noted that, despite being a platform of American origin, as has already been said before, its streamers were mostly Russian and Central European (so many people believed that it was a Russian application); however, Periscope's audience was ostensibly international. We speak in the past tense because Periscope is in low hours, and it is very difficult for it to be able to rebuild; most likely, it will not take long to disappear completely, due to the migration of its users to other platforms. The causes are the usual ones, perhaps, in this case, with the addition of the "Russian take" (by the streamers) of the platform.

\section{Audience and Fans}

Here we proceed to a very summary description of the type of user that has been observed in each of the studied platforms. In a later article, this typology will be analysed in depth with the information obtained based on the empirical information that can be extracted from certain forums frequented by the most "recalcitrant" fans of this types of eroticism.

Youtube: followers of the type of channel studied can be classified into several groups: general public (curious and fans), owners of similar channels, fans of tween and teen eroticism and people with undefined sexual identity.

Stickam: first of all the curious (who, generally, stayed); there were also unconditional fans of some of the "divas" of Stickam, for whom they felt an absolute devotion; the bulk of users was moving between channels; among the users existed the "cappers", who were dedicated to recording streams, because many of the streamers did not keep their recordings. The streamers also had the option of doing their performance for a fan or a group of trusted fans (the "privates"). The so-called White knights began to appear, a kind of censorship sect that is dedicated to harassing and frightening streamers (in fact, it is said that there was a case of suicide due to the enactment of one of them).

Periscope: a part of the followers of the streams on this platform was made up of ex-Stickam users; they were surprised that on this North American platform, the streamers, for the most part, only spoke Russian. This meant 
that to interact with the streamers they had to do it through a translator (like Google Translator); the precariousness of the translations could give rise to misunderstandings that could cause the expulsion of the user by the owner of the channel. Only Russian-speakers could relate to streamers in the same way that Stickam had done, although times were already changing for this type of interaction, and new applications exclusively for mobile devices were already looming on the horizon. On the other hand, the sect of the White knights was increasing its attacks.

As had been the case in Stickam, in Periscope certain streamers got naked, and some of them carried out erotic (or, if preferred, "sexual") games. This type of performance attracted all types of audiences from all countries of the world (there were streams that had exceeded 100,000 viewers in a matter of minutes).

\section{Forums}

In a future article we will deal with, as mentioned above, "recalcitrant users". They usually visit certain types of ad hoc forums. Here we proceed to a very summary representation of the phenomenon.

Because of the interest generated by the phenomenon, forums about Stickam, Periscope and other platforms emerged on the Internet. In the Stickam forums they talked, above all, about famous streamers and could also be seen "walls" with requests (like that of some famous stream in particular that some fans missed). In the most general forums there was everything: those who spoke in chats about any current or personal topic; those who talked about matters related to eroticism; those who were desperately looking for a video of the divas who were the object of their devotion; and also, as might be expected, those who asked for or offered what they called "CP", an acronym for child pornography (it can be inferred, consequently, that for them the streams itself were not pornographic).

Since the last statement in the previous paragraph is not trivial, a review of the concepts (with which we judge reality) that we take for granted cannot be postponed. From our point of view, built through the analysis of the uses of language by politicians, journalists and specialists of all kinds, there is no clear definition of what child pornography consists of (as happens with the concepts of sex and pornography), so that, depending on who deals with the matter, it may fit within the concept what the person who handles it seems appropriate. We think that, for the sake of accuracy, the concept should be narrowed down and limited to its analogue with adult pornography: that is, child pornography would be that type of production that involves a team of adults performing the same functions as in "normal pornography": producers, scriptwriters, directors, cameramen, actors, etc. We consider that it is an error to qualify as "child pornography" a recording, for example, carried out by an adolescent while performing erotic acts, since this necessarily entails (yet there is no pornography without "actors") the qualification of the protagonist of said recording as a "porn actress", which is, clearly, nonsense. It is not possible to equate, without committing a terrible fallacy, a pornographic production carried out by adults using sexually minors for illicit purposes with a home video or a stream freely produced by an adolescent. Consequently, for the sake of terminological clarity, the well-being of minors and the mental health of citizens, it is absolutely necessary to carry out a rigorous and openminded analysis of this phenomenon, to elucidate under which concept each specific aspect can be subsumed and, if necessary, create new concepts for what is not subsumed in the existing ones [12-16].

\section{Conclusion}

The analysed facts and processes certify the thesis held by the most advanced researchers in this matter: that the tweens and the teens (for calling the pre-adult stage somehow) not only seek and obtain on the Internet the information they need about this conceptual magma to which we refer to as "sexuality", but they also use the internet as a medium or context through which to express themselves erotically (that is, to show and contrast their own eroticism) and, consequently, to develop their own sexual identity, thereby contributing to the development of own personal identity (since the former is part of it). Thus, from the studies that show that people who have had more sexual freedom in the progressive stages of development enjoy greater personal fulfilment in adulthood, it can be inferred that this also applies to the Internet age, which is ours' (and even more so that of its natives): the more freedom, the more fullness.

Despite what has been stated up to this point, we still have to ask ourselves about the attitude of one of the main actors in this whole phenomenon. What, then, has been the reaction of society (both families in general and public institutions) to these phenomena? As was foreseeable, the social reaction has always been the same as it has always had when facing what is either unknown or feared (or both) and which, supposedly, violates established "uses and traditions": intolerance. Instead of creating a happy and safe environment so that it is possible to freely express one's eroticism and to develop unhindered sexual identity (and, therefore, one's own personal identity), what has been done has been the opposite: to implement a whole series of inquisitorial measures, more typical of immature societies with a foolish idiosyncrasy than of our supposedly democratic and advanced societies. However, we venture to predict that, in the same way that society has had to end up accepting 
homosexuality, lesbianism, and, in general, total freedom of choice regarding sexual identity (the opposite, today, already is "politically incorrect"), will have to accept, sooner or later, that adolescents and pre-adolescents also have the right to have their rights and freedoms in this matter (excuse the intentional redundancy), and, consequently, society will have to abandon their reservations regarding this phenomenon and must integrate the new forms of expression of eroticism within the increasingly comprehensive version that we have of normality.

Those who see the "evil" in adolescent erotic free expression are not aware that they are anchored in the past ... well, as Karl Marx ironically said in his foreword to Das Kapital "Le mort saisit le vif!" (The dead clings to the living). It is not acceptable that prejudices and common places are used, as if they were thorough concepts, unappealable truths or valid arguments, in the analysis of these complex processes, an analysis that should be carried out meticulously, applying scientific criteria in a rigorous way, especially when it is about the integral development of human life. We have already seen what is achieved with such attitudes: exactly the opposite of what is supposedly intended. Instead of trying to analyse a fact that "cannot be accepted" or, rather, one does not know how to accept (as in his time it happened with homosexuality and a too long etcetera), at no time do those who apply these criteria raise the possibility that they are wrong and, nor does it occur to them to try to find out if their approach to the matter is the most suitable, to be able to treat it as an object of research apart from what we can qualify as their "internal demons" (in the sense that they are alien to the thing itself); on the contrary, from what it seems society has allowed itself to be carried away by these demons and has left the adolescent and pre-adolescent population defenceless before them, without calculating the possible psychosociological risks that this "demonization" may cause or the drift that the phenomenon can take. In fact, this censorship prevention has been the cause of the repression on YouTube and the closure of Stickam and other conventional platforms, which has had the effect of displacing the phenomenon towards more uncontrollable platforms (such as Único or Snapchat) that have a "specialized" and recalcitrant audience, for which nudity is no longer an option, but an obligation and the typical video of such applications is closer to a visit to the gynaecologist than to a more or less erotic Stickam's stream.

Note: it was our intention to elaborate a detailed analysis of the empirical material, to organize it in categories; establish common patterns and differences; observe all the processes that could provide useful information for researchers specialized in the subject; share it with interested colleagues to verify hypotheses and obtain feedback based on material observation; increase the degree of scientific solidity of the research and, finally, have a stock of material samples of the object of study. Unfortunately, the administration has seized the empirical material that supports this work of which the return has been requested.

\section{References}

1. Li Gu (2020) Sexuality Development in Childhood. New York University Shanghai.

2. David S, Kaveri SM (2014) Adolescent Sexuality on the Internet: A Developmental Perspective. Oxford University Press.

3. Bunge M (2012) Materia y mente. Gedisa.

4. Freud S (2011) Psicopatología de la vida cotidiana. AE.

5. Lorah DD, Frank BM (2011) Puberty and Its Measurement: A Decade in Review. Journal of Research on Adolescence 21(1): 180-195.

6. Sperling MA (2014) Pediatric endocrinology, $4^{\text {th }}$ (Edn.), Elsevier Health Sciences.

7. Catrin B, Elisabet HN, Christina S, Tanja T, Stavros I (2021) Changes in sexual behavior among highschool students over a 40year period. Scientific Reports 11: 13963.

8. Arbeit Miriam R (2014) What Does Healthy Sex Look Like among Youth? Towards a Skills-Based Model for Promoting Adolescent Sexuality Development. Human Development 57: 259-286.

9. Bair Steele M (2014) Do These Norms Make Me Look Fat? The Effect of Exposure to Others' Body Preferences on Personal Body Ideals. Body Image 11(3): 275-281.

10. English Wikipedia. Articles (YouTube, Stickam, Periscope).

11. Gözde Y (2016) Entertainment Industry versus Culture Industry: Designers of Consumption Society. TRTAkademi 1(1): 231-245.

12. (2019) International Journal of Advanced Studies in Sexology.

13. Mtandika N (2013) The Role of Entertainment Television in Shaping Social Behaviour of Children. A case of Kinondoni Municipality in Dar Es Salaam Region. Open University of Tanzania.

14. Marx K (1976) Das Kapital. OME 45. Grijalbo.

15. Norris Anne E, Smith AU, Ferranti D, Choi HJ (2021) The Measurement of Female Early Adolescent Sexual Desire. 
J Sex Res 59(1): 69-84.

16. VanderLaan DP, Wong WI (2020) Gender and sexuality development: Contemporary theory and research. Springer. 